Published in final edited form as:

Clin Rheumatol. 2013 May ; 32(5): 617-621. doi:10.1007/s10067-012-2145-7.

\title{
Clinical and serological features of systemic sclerosis in a Chinese cohort
}

\author{
Jiucun Wang, \\ Ministry of Education (MOE) Key Laboratory of Contemporary Anthropology and State Key \\ Laboratory of Genetic Engineering, School of Life Sciences, Fudan University, Shanghai, China \\ Institute of Rheumatology, Immunology and Allergy, Fudan University, Shanghai, China \\ Shervin Assassi, \\ The University of Texas Medical School at Houston, 6431 Fannin, MSB5.270, Houston, TX \\ 77030, USA \\ Gang Guo, \\ Yiling Hospital, Shijiazhuang, Hebei Province, China \\ Wenzhen Tu, \\ Shanghai Traditional Chinese Medicine-Integrated Hospital, Shanghai, China \\ Wenyu Wu, \\ Division of Dermatology, Huashan Hospital, Fudan University, Shanghai, China \\ Institute of Rheumatology, Immunology and Allergy, Fudan University, Shanghai, China
}

Li Yang,

Division of Rheumatology, Teaching Hospital of Chengdu University of TCM, Chengdu, Sichuan

Province, China

\section{Rong Xiao,}

Department of Dermatology, Second Xiangya Hospital, Central South University, Changsha, Hunan Province, China

\section{Yongqiang Zhao,}

Yiling Hospital, Shijiazhuang, Hebei Province, China

\section{Haiyan Chu,}

Ministry of Education (MOE) Key Laboratory of Contemporary Anthropology and State Key Laboratory of Genetic Engineering, School of Life Sciences, Fudan University, Shanghai, China

Jie Liu,

Ministry of Education (MOE) Key Laboratory of Contemporary Anthropology and State Key Laboratory of Genetic Engineering, School of Life Sciences, Fudan University, Shanghai, China

\section{Yanyun Ma,}

Ministry of Education (MOE) Key Laboratory of Contemporary Anthropology and State Key Laboratory of Genetic Engineering, School of Life Sciences, Fudan University, Shanghai, China

Sarah J. Kim,

The University of Texas Medical School at Houston, 6431 Fannin, MSB5.270, Houston, TX 77030, USA

\footnotetext{
(C) Clinical Rheumatology 2012

Correspondence to: Xiaodong Zhou, xiaodong . zhou@uth . tmc . edu.

Disclosures None.
} 
Li Jin,

Ministry of Education (MOE) Key Laboratory of Contemporary Anthropology and State Key Laboratory of Genetic Engineering, School of Life Sciences, Fudan University, Shanghai, China

Maureen D. Mayes,

The University of Texas Medical School at Houston, 6431 Fannin, MSB5.270, Houston, TX 77030, USA

Hejian Zou, and

Division of Rheumatology, Huashan Hospital, Fudan University, Shanghai, China

Institute of Rheumatology, Immunology and Allergy, Fudan University, Shanghai, China

Xiaodong Zhou

The University of Texas Medical School at Houston, 6431 Fannin, MSB5.270, Houston, TX 77030, USA

Hejian Zou: hejianzou@gmail.com; Xiaodong Zhou: xiaodong.zhou@uth.tmc.edu

\section{Abstract}

Our goal was to study the prevalence of systemic sclerosis (SSc) subtypes, autoantibody profile, and pulmonary fibrosis in a large group of Han Chinese. Chinese SSc patients $(n=419)$ were recruited from a multicenter study including hospitals and outpatient clinics in China. All patients met the American College of Rheumatology classification criteria for SSc. Anti-topoisomerase (ATA), anti-centromere (ACA), anti- RNA polymerase III (anti-RNAP3), and anti-U1ribonucleoprotein (anti-U1RNP) were detected utilizing commercially available kits. The clinical and autoantibody information in Chinese patients was compared to that in the US Caucasian patients ( $n=834)$, recruited from the Genetics versus Environment in Scleroderma Outcome Study and Scleroderma Family Registry. Chi-square test was utilized for the abovementioned comparisons. Chinese patients showed $40.3 \%$ limited (lcSSc) and $59.7 \%$ diffuse (dcSSc) forms of SSc. ATA was found in 59.9\%, ACA in $13.4 \%$, anti-RNAP3 in $1.3 \%$, and anti-U1RNP in 18 $\%$ of Chinese SSc patients. Compared to US patients (65.1\% lcSSc, $34.9 \%$ dcSSc, ATA in 18.7 $\%$, ACA in $32.4 \%$, anti-RNAP3 in $17.4 \%$, and anti-U1RNP in $2.8 \%$ ), Chinese SSc patients are significantly higher in dcSSc and the frequencies of ATA and anti-U1RNP, but lower in ACA and anti-RNAP3. In addition, pulmonary fibrosis was observed in $78 \%$ Chinese SSc patients and was strongly associated with the presence of ATA. The present study represents the first report of SSc features in a large group of Chinese patients. Clinical subtypes and the frequencies of SSc-related autoantibodies in Chinese SSc patients are significantly different from those in SSc patients of the US Caucasian descent.

\section{Keywords}

Anti-centromere antibody; Anti-topoisomerase antibody; Autoantibodies; Pulmonary fibrosis; Systemic sclerosis

\section{Introduction}

Systemic sclerosis ( $\mathrm{SSc}$ ) or scleroderma is a rare and complex autoimmune disease. It is clinically classified into two subsets: limited cutaneous (lcSSc) and diffuse cutaneous $(\mathrm{dcSSc}) \mathrm{SSc}$. The latter subset is characterized by more rapid progression of skin and visceral involvement as well as poorer prognosis [1]. SSc also can be subgrouped by autoantibody subsets. The most common of these autoantibodies are directed against DNA topoisomerase I (ATA), centromeric proteins (ACA), and RNA polymerases III (antiRNAP3), as well as other less commonly occurring autoantibodies including U1- 
ribonucleoprotein (anti-U1RNP) [1]. Individual SSc patients rarely have more than one of these autoantibodies, and each autoantibody subset is associated with certain disease features [2]. Previously, several studies suggested that the prevalence of SSc subsets varies in different ethnic groups. For instance, the patients of Choctaw Native American and African American descent are more likely to develop dcSSc compared to the patients of European descent [3, 4]. Furthermore, Choctaw Native Americans, African Americans, Koreans, and Thais have relatively higher frequencies of ATA [3-6]. Although Han Chinese is a large population, only one report on Chinese SSc with a small sample size was previously reported [7]. Recently, we established a multicenter SSc consortium in China under the International Network of Scleroderma Clinical Care and Research (http:// www.inscar-global.org). The goal of the present study is to investigate the prevalence of SSc disease subtypes, autoantibody profile, and organ involvement in a large group of Han Chinese patients with SSc.

\section{Materials and methods}

\section{Patient enrollment}

Chinese patients were recruited from a multicenter study including hospitals and outpatient clinics in Shanghai, Hebei Province, Sichuan Province, and Hunan Province in China. Patients either met the American College of Rheumatology classification criteria for SSc (96.1\% of Chinese patients) [8] or had at least three out of five CREST features (calcinosis, Raynaud's phenomenon, esophageal dysmotility, sclerodactyly, and telangiectasia) with sclerodactyly being mandatory (3.9\%) [9]. Mean duration of the disease was 7.3 years ranging from 1 to 32 years (from the first non-Raynaud's phenomenon symptom). Diagnosis of pulmonary fibrosis was confirmed with either chest X-ray (41.9\%) or thorax CT (58.1\%) in different hospitals. The US Caucasian patients $(n=834)$ were recruited from the Genetics versus Environment in Scleroderma Outcome Study and Scleroderma Family Registry [10]. The ethical approvals of the studies were obtained from each institutional review board.

\section{Autoantibody tests}

Patient's sera were tested for antinuclear antibodies (ANA) by indirect immunofluorescence using HEp-2 cells as antigen substrate (Antibodies, Davis, CA, USA). ATA and antiU1RNP were detected by passive immunodiffusion against calf thymus extracts (INOVA Diagnostics). ACA was determined by indirect immunofluorescence using HEp-2 cells. Anti-RNAP3 was detected utilizing commercially available kits (NBL, Nagoya, Japan).

\section{Statistical analysis}

Chi-square test was utilized for the abovementioned comparisons. The $p$ values less than 0.05 were considered statistically significant. The statistical program Epi Info ${ }^{\mathrm{TM}} 3.5 .4$ was used in the analysis.

\section{Results}

A total of 419 Han Chinese SSc patients were enrolled in the studies. The female-to-male ratio was 4.9:1. Clinical classification of these patients showed that there were 169 (40.3\%) limited and $250(59.7 \%)$ diffuse forms of SSc. Male and female patients did not differ in frequency of $1 c S S c$ versus dcSSc $(p=0.65)$.

All Chinese patients were examined for ATA and ACA. In addition, 303 Chinese consecutive patients were assayed for anti-RNAP3 and 311 for anti-U1RNP. ATAwas found in $251(59.9 \%)$, ACA in $56(13.4 \%)$, anti-RNAP3 in $4(1.3 \%)$, and anti-U1RNP in 56 (18 $\%)$ patients, while $90.7 \%$ of patients were ANA positive. 
The comparison of Chinese SSc patients to 834 SSc patients of self-reported European decent (US Caucasian) is shown in Table 1. ATA, ACA, anti-RNAP3, and anti-U1RNP were observed in $156(18.7 \%), 270$ (32.4\%), 145 (17.4\%), and 23 (2.8\%) US Caucasian patients, respectively. The frequencies of ATA and anti-U1RNP were significantly higher among Chinese patients, while the ACA and anti-RNAP3 were more prevalent among US Caucasian patients. Chinese patients also had a higher proportion of the dcSSc subtype than US Caucasian patients (60 versus $35 \%$ ).

The associations of these autoantibodies with clinical subtypes of Chinese SSc are shown in Table 2. Among 251 ATA-positive SSc patients, there were 161 with dcSSc $(64.1 \%)$ and 90 with lcSSc (35.9\%) compared with 168 ATA-negative patients of whom 89 had dcSSc (53 $\%)$ and 79 had lcSSc $(47 \%)$. ATA was associated with dcSSc in Chinese patients $(p=0.029$, odds ratio $(\mathrm{OR})=1.59)$. SSc patients with ACA, anti-RNAP3, and anti-U1RNP did not show association with either dcSSc or lcSSc $(p>0.05)$.

There were 328 Chinese patients examined with chest X-ray and/or thorax CT for diagnosis of pulmonary fibrosis. The associations of pulmonary fibrosis with the presence of specific autoantibodies in Chinese patients are shown in Table 3. Pulmonary fibrosis was observed in 161 of 187 ATA-positive and in 95 of 141 ATA-negative patients, indicating a strong association of ATA with pulmonary fibrosis $\left(p=8.84 \times 10^{-5}, \mathrm{OR}=3.0\right)$. In contrast, it was present in 23 of 44 ACA-positive and 233 of 284 ACA-negative patients, indicating a negative association of ACA with pulmonary fibrosis $\left(p=2.2 \times 10^{-5}, \mathrm{OR}=0.24\right)$. In addition, pulmonary fibrosis showed a marginal association with dcSSc (in $81.64 \% \mathrm{dcSSc}$ versus $71.9 \%$ lcSSc, $p=0.055, \mathrm{OR}=1.74)$, but no association with anti-RNAP3 and anti-U1RNP auto-antibodies $(p>0.05)$.

\section{Discussion}

The present study represents the largest report on the SSc-related autoantibodies and clinical subtypes in patients of Han Chinese decent. Herein, we demonstrated for the first time that Chinese SSc patients appeared similar to patients of European descent in gender distribution and ANA positivity, but differ in the proportion of clinical subsets and frequencies of SScrelated autoantibodies.

A higher proportion of dcSSc and pulmonary fibrosis was observed in the Chinese cohort compared to the US cohort. This observation is similar to that in SSc patients of Choctaw Native American and African American descent [3, 4].

The differences in frequency of SSc-related autoantibodies between Han Chinese and US Caucasian patients were significant in all four autoantibodies examined. A higher frequency of ATA $(59.9 \%)$ and anti-U1RNP (18\%), but a lower occurrence of ACA (13.4\%) and anti-RNAP3 $(1.3 \%)$, was observed in Chinese SSc patients. It is note-worthy that a previous report of $36 \mathrm{SSc}$ patients of Chinese descent showed that ATA and anti-RNP antibodies were present in 48.4 and $17.2 \%$, respectively [6], paralleling the autoantibody frequencies observed in the present study. In addition, the frequencies of ATA and ACA in Chinese SSc are very close to a previous report in Korean SSc patients (62.2\% ATA and $12.03 \%$ ACA) [4]. Moreover, a high frequency of ATA (76\%) and a low frequency of ACA (2\%) also were reported in SSc patients of Thai decent [5]. These data indicate a high frequency of ATA and a low frequency of ACA positivity among Asian SSc patients.

Similar to the findings in other ethnic populations $[2,9,10]$, the presence of ATA in Chinese SSc patients was strongly associated with dcSSc and pulmonary fibrosis, while ACA was correlated with less pulmonary involvement (Table 3). On the other hand, in addition to a higher proportion of dcSSc in ATA-positive patients (64.1\%), ACA-positive Chinese 
patients also have a relatively high occurrence of dcSSc (55.4\%), which appeared unique to this Chinese cohort.

A markedly low frequency of anti-RANP3 (1.3\%) among Chinese SSc patients is a novel finding. There were also reports of low prevalence of anti-RANP3 in Japanese and European populations including French and Italian (4, 9.1, and $8 \%$, respectively) [11-13].

Considering that RNAP3 is strongly associated with scleroderma renal crisis [14], we expect that Chinese SSc patients have a low prevalence of this disease manifestation.

The frequency of anti-U1RNP observed in the Chinese cohort (18\%) is significantly higher than that in the US cohort ( $2.8 \%)$. Neither clinical subtypes nor pulmonary fibrosis was associated with the presence of this antibody in any of the two cohorts. Interestingly, a higher frequency of anti-U1RNP (30\%) also was reported in SSc patients of African American population [15]. We do not believe that the observed higher frequency of patients with dcSSc and ATA among Chinese patients is secondary to referral bias because patients were recruited from a wide geographic area that included inpatient and outpatient clinics.

In summary, the present study represents the first report of clinical subtypes and autoantibodies of SSc in a large sample of Chinese patients. We demonstrate that the frequencies of SSc-related autoantibodies and clinical subtypes in Chinese patients with SSc are significantly different from US Caucasian SSc patients in the USA. The autoantibody profile among Chinese patients with SSc is similar to that among other Asian populations. These findings suggest that SSc patients in various geographic areas differ in their serological and clinical manifestations.

\section{Acknowledgments}

This study was supported by NIH/NIAID-1U01AI09090-01, NIH/NIAMS-R01-AR055258, P50-AR-054144, K23AR-061436, the Major National Science and Technology Program of China (grant number 2008ZX10002-002), the Science and Technology Committee of Shanghai Municipality (11410701800 and 10JC1402100), and the National Natural Science Foundation of China (grant number 30971594).

\section{References}

1. Bunn CC, Black CM. Systemic sclerosis: an autoantibody mosaic. Clin Exp Immunol. 1999; 117:207. [PubMed: 10444248]

2. Steen VD. Autoantibodies in systemic sclerosis. Semin Arthritis Rheum. 2005; 35:35-42. [PubMed: 16084222]

3. Arnett FC, Howard RF, Tan F, Moulds JM, Bias WB, Durban E, et al. Increased prevalence of systemic sclerosis in a Native American tribe in Oklahoma. Association with an Amerindian HLA haplotype. Arthritis Rheum. 1996; 39:1362-1370. [PubMed: 8702445]

4. Steen V, Domsic RT, Lucas M, Fertig N, Medsger TA. A clinical and serologic comparison of African American and Caucasian patients with systemic sclerosis. Arthritis Rheum. 2012

5. Zhou X, Lee JE, Arnett FC, Xiong M, Park MY, Yoo YK, et al. HLA-DPB1 and DPB2 are genetic loci for systemic sclerosis: a genome-wide association study in Koreans with replication in North Americans. Arthritis Rheum. 2009; 60:3807-3814. [PubMed: 19950302]

6. McNeilage LJ, Youngchaiyud U, Whittingham S. Racial differences in antinuclear antibody patterns and clinical manifestations of scleroderma. Arthritis Rheum. 1989; 32:54-60. [PubMed: 2783552]

7. Low AH, Johnson SR, Lee P. Ethnic influence on disease manifestations and autoantibodies in Chinese-descent patients with systemic sclerosis. J Rheumatol. 2009; 36:787-793. [PubMed: 19228652]

8. Subcommittee for Scleroderma Criteria of the American Rheumatism Association Diagnostic and Therapeutic Criteria Committee. Preliminary criteria for the classification of systemic sclerosis (scleroderma). Arthritis Rheum. 1980; 23:581-590. [PubMed: 7378088] 
9. LeRoy EC, Black C, Fleischmajer R, Jablonska S, Krieg T, Medsger TA Jr, et al. Scleroderma (systemic sclerosis): classification, subsets and pathogenesis. J Rheumatol. 1988; 15:202-205. [PubMed: 3361530]

10. Assassi S, Sharif R, Lasky RE, McNearney TA, Estrada-Y-Martin RM, Draeger H, et al. Predictors of interstitial lung disease in early systemic sclerosis: a prospective longitudinal study of the GENISOS cohort. Arthritis Res Ther. 2010; 12:R166. [PubMed: 20813056]

11. Fritzler MJ, Ayer LM. Anticentromere antibodies: clinical and biological significance. J Rheumatol. 1982; 9:489-490. [PubMed: 6982333]

12. Hamaguchi Y, Hasegawa M, Fujimoto M, Matsushita T, Komura K, Kaji K, et al. The clinical relevance of serum antinuclear antibodies in Japanese patients with systemic sclerosis. Br J Dermatol. 2008; 158:487-495. [PubMed: 18205876]

13. Meyer O, De Chaisemartin L, Nicaise-Roland P, Cabane J, Tubach F, Dieude P, et al. Anti-RNA polymerase III antibody prevalence and associated clinical manifestations in a large series of French patients with systemic sclerosis: a cross-sectional study. J Rheumatol. 2010; 37:125-130. [PubMed: 19918031]

14. Bardoni A, Rossi P, Salvini R, Bobbio-Pallavicini F, Caporali R, Montecucco C. Autoantibodies to RNA-polymerases in Italian patients with systemic sclerosis. Clin Exp Rheumatol. 2003; 21:301306. [PubMed: 12846047]

15. Krzyszczak ME, Li Y, Ross SJ, Ceribelli A, Chan EK, Bubb MR, et al. Gender and ethnicity differences in the prevalence of scleroderma-related autoantibodies. Clin Rheumatol. 2011; 30:1333-1339. [PubMed: 21523365] 
